Constructive Algebra and Systems Theory

B. Hanzon and M. Hazewinkel (Editors)

Royal Netherlands Academy of Arts and Sciences, 2006

\title{
The Cartan covering and complete integrability of the KdV-mKdV
}

\section{system}

\author{
P.H.M. Kersten ${ }^{a}$ and I.S. Krasil'shchik ${ }^{b}$ \\ ${ }^{a}$ University of Twente, Faculty of Mathematical Sciences, P.O. Box 217, 7500 AE Enschede, \\ The Netherlands \\ ${ }^{\mathrm{b}}$ Independent University of Moscow, B. Vlasevsky 11, 119002 Moscow, Russia \\ E-mail: kersten@math.utwente.nl (P.H.M. Kersten)
}

\section{ABSTRACT}

The coupled $\mathrm{KdV}-\mathrm{mKdV}$ system arises as the classical part of one of superextensions of the $\mathrm{KdV}$ equation. For this system, we prove its complete integrability, i.e., existence of a recursion operator and of infinite series of symmetries. After giving a short introduction into the theory of symmetries, coverings, and the notion of Cartan-covering, the recursion operator will be constructed as a symmetry in the Cartan covering of the $\mathrm{KdV}-\mathrm{mKdV}$ system.

\section{INTRODUCTION}

There are several supersymmetric extensions of the classical Korteweg-de Vries equation $(\mathrm{KdV})[6,9,10]$. One of them is of the form (the so-called $N=2, A=1$ extension [2])

$$
\begin{aligned}
u_{t}= & -u_{3}+6 u u_{1}-3 \varphi \varphi_{2}-3 \psi \psi_{2}-3 w w_{3}-3 w_{1} w_{2}+3 u_{1} w^{2}+6 u w w_{1} \\
& +6 \psi \varphi_{1} w-6 \varphi \psi_{1} w-6 \varphi \psi w_{1} \\
\varphi_{t}= & -\varphi_{3}+3 \varphi u_{1}+3 \varphi_{1} u-3 \psi_{2} w-3 \psi_{1} w_{1}+3 \varphi_{1} w^{2}+6 \varphi w w_{1} \\
\psi_{t}= & -\psi_{3}+3 \psi u_{1}+3 \psi_{1} u+3 \varphi_{2} w+3 \varphi_{1} w_{1}+3 \psi_{1} w^{2}+6 \psi w w_{1} \\
w_{t}= & -w_{3}+3 w^{2} w_{1}+3 u w_{1}+3 u_{1} w
\end{aligned}
$$

where $u$ and $w$ are classical (even) independent variables while $\varphi$ and $\psi$ are odd ones (here and below the numerical subscript at an unknown variable denotes its

Key words and phrases: Coupled KdV-mKdV system, Cartan-covering, Complete integrability, Recursion operators, Symmetries, Conservation laws, Coverings, Deformations, Superdifferential equations 
derivative over $x$ of the corresponding order). Being completely integrable itself, this system gives rise to an interesting system of even equations

$$
\begin{aligned}
& u_{t}=-u_{3}+6 u u_{1}-3 w w_{3}-3 w_{1} w_{2}+3 u_{1} w^{2}+6 u w w_{1} \\
& w_{t}=-w_{3}+3 w^{2} w_{1}+3 u w_{1}+3 u_{1} w
\end{aligned}
$$

which can be considered as a sort of coupling between the $\mathrm{KdV}$ (with respect to $u$ ) and the modified $\mathrm{KdV}$ (with respect to $w$ ) equations. In fact, setting $w=0$, we obtain

$$
u_{t}=-u_{3}+6 u u_{1}
$$

while for $u=0$ we have

$$
w_{t}=-w_{3}+3 w^{2} w_{1}
$$

The above indicates why we call (1) the KdV-mKdV system.

In what follows, we prove complete integrability, cf. [1], of system (1) by establishing existence of infinite series of symmetries and/or conservation laws. Toward this end we construct a recursion operator using the techniques of deformation theory introduced in [5] and extensively described and exemplified in [6].

In practical situations the construction of a deformation of the equation structure boils down to the construction of symmetries in an augmented setting of the equation or system at hand.

In Section 1 of this lecture we shall set out the nonlocal setting for differential equations and describe the notion of nonlocal symmetries in this setting.

Section 2 deals with a particular type of nonlocality, the Cartan covering of an equation.

Section 3 combines the two previous types of coverings, and it is this covering where the recursion operator for symmetries is obtained as a symmetry in this covering.

In these first three sections the classical $\mathrm{KdV}$ equations acts as the main example. Finally in Section 4 we shall present symmetries, conservation laws, nonlocalities and the recursion operator for symmetries for the coupled $\mathrm{KdV}-\mathrm{mKdV}$ system (1).

\section{NONLOCAL SETTING FOR DIFFERENTIAL EQUATIONS}

As standard example, to illustrate the notions, we take KdV-equation

$$
u_{t}=u u_{x}+u_{x x x} .
$$

We consider $Y \subset J^{\infty}(x, t ; u)$ the infinite prolongation of (2), cf. [7,8], where coordinates in the infinite jet bundle $J^{\infty}(x, t ; u)$ are given by $\left(x, t, u, u_{x}, u_{t}, \ldots\right)$ and $Y$ is formally described as the submanifold of $J^{\infty}(x, t ; u)$ defined by 
(3)

$$
\begin{aligned}
& u_{t}=u u_{x}+u_{x x x}, \\
& u_{x t}=u u_{x x}+u_{x}^{2}+u_{x x x x},
\end{aligned}
$$

As internal coordinates in $Y$ one chooses $\left(x, t, u, u_{x}, u_{x x}, \ldots\right)$ while $u_{t}, u_{x t}, \ldots$ are obtained from (3).

The Cartan distribution on $Y$ is given by the total partial derivative vector fields

$$
\widetilde{D}_{x}=\partial_{x}+\sum_{n \geqslant 0} u_{n+1} \partial_{u_{n}},
$$

$$
\widetilde{D}_{t}=\partial_{t}+\sum_{n \geqslant 0} u_{n t} \partial_{u_{n}},
$$

where $u_{1}=u_{x}, u_{2}=u_{x x}, \ldots ; u_{1 t}=u_{x t} ; u_{2 t}=u_{x x t}, \ldots$.

Classically the notion of a generalized or higher symmetry of a differential equation $F=0$ is defined as a vertical vector field $V$

$$
V=\vartheta_{f}=f \partial_{u}+\widetilde{D}_{x}(f) \partial_{u_{1}}+\widetilde{D}_{x}^{2}(f) \partial_{u_{2}}+\cdots,
$$

where $f \in C^{\infty}(Y)$ such that,

$$
\ell_{F}(f)=0
$$

where in (6) $\ell_{F}$ is the universal linearisation operator $[11,7]$ which reads in the case of $\mathrm{KdV}$-equation (3)

$$
\widetilde{D}_{t}(f)-\widetilde{D}_{x}(f)-u_{1} \cdot f-\left(\widetilde{D}_{x}\right)^{3}(f)=0 .
$$

Let now $W \subset \mathbb{R}^{m}$ with coordinates $\left(w_{1}, \ldots, w_{m}\right)$.

The Cartan distribution on $Y \otimes W$ is given by

$$
\begin{aligned}
& \bar{D}_{x}=\widetilde{D}_{x}+\sum_{j=1}^{m} X^{j} \frac{\partial}{\partial w_{j}}, \\
& \bar{D}_{t}=\widetilde{D}_{t}+\sum_{j=1}^{m} T^{j} \partial_{w_{j}}
\end{aligned}
$$

where $X^{j}, T^{j} \in C^{\infty}(Y \otimes W)$ such that

$$
\left[\bar{D}_{x}, \bar{D}_{t}\right]=0
$$

which yields the so-called covering condition

$$
D_{x}(T)-D_{t}(X)+[X, T]=0
$$


whereas in (9) $\left[{ }^{*},{ }^{*}\right]$ is the Lie bracket for vector fields $X=\sum_{j=1}^{m} X^{j} \partial_{w_{j}}, T=$ $\sum_{j=1}^{m} T^{j} \partial_{w_{j}}$ defined on $W$.

A nonlocal symmetry is a vertical vector field on $Y \otimes W$, i.e., of the form (5), which satisfies $\left(f \in C^{\infty}(Y \otimes W)\right)$

$$
\bar{\ell}_{F}(f)=0
$$

which for $\mathrm{KdV}$ results in

$$
\bar{D}_{t}(f)-u \bar{D}_{x}(f)-u_{1} f-\left(\bar{D}_{x}\right)^{3}(f)=0 .
$$

Formally this is just what is called the shadow of the symmetry, i.e., not bothering about the $\partial_{w^{j}}, j=1, \ldots, m$, components.

The construction of the associated $\partial_{w^{j}}, j=1, \ldots, m$, components is called the reconstruction problem [4]. For reasons of simplicity, we omit this reconstruction problem, i.e., reconstructing the vector field from its shadow.

The classical Lenard recursion operator $\mathcal{R}$ for $\mathrm{KdV}$ equation,

$$
\mathcal{R}=D_{x}^{2}+\frac{2}{3} u+\frac{1}{3} u_{1} D_{x}^{-1}
$$

which is just such, that

$$
\begin{aligned}
& f_{0}=u_{1}, \\
& \mathcal{R} f_{0}=f_{1}=u u_{1}+u_{3}, \\
& \mathcal{R} f_{1}=f_{2}=u_{5}+\frac{5}{3} u_{3} u+\frac{10}{3} u_{2} u_{1}+\frac{5}{6} u_{1} u^{2},
\end{aligned}
$$

i.e., creating the $(x, t)$-independent hierarchy of higher symmetries, has an action on vertical symmetry $\vartheta_{\bar{f}_{-1}}$ (Gallilei-boost)

$$
\begin{aligned}
& \bar{f}_{-1}=\left(1+t u_{1}\right) / 3 \\
& \mathcal{R} \bar{f}_{-1}=\bar{f}_{0}=2 u+x u_{1}+3 t\left(u_{3}+u u_{1}\right), \\
& \bar{f}_{1}=\mathcal{R} \bar{f}_{0}=3 t\left(f_{2}\right)+x\left(f_{0}\right)+4 u_{2}+\frac{4}{3} u^{2}+\frac{1}{3} u_{1} D_{x}^{-1}(u) .
\end{aligned}
$$

If we introduce the variable $p\left(=w_{1}\right)$ through

$$
\begin{aligned}
& p_{x}=u, \\
& p_{t}=u_{2}+\frac{1}{2} u^{2}, \\
& \text { i.e., } \quad D_{t}(u)=D_{x}\left(u_{2}+\frac{1}{2} u^{2}\right)
\end{aligned}
$$

then $\vartheta_{\bar{f}_{1}}$ is the shadow of a nonlocal symmetry in the one-dimensional covering of $\mathrm{KdV}$-equation by

$$
p=w_{1}, \quad X_{1}=u, \quad T_{1}=u_{2}+\frac{1}{2} u^{2} .
$$


So, by its action the Lenard recursion operator creates nonlocal symmetries in a natural way.

More applications of nonlocal symmetries can be found in, e.g., [6].

\section{A SPECIAL TYPE OF COVERING: THE CARTAN-COVERING}

We discuss a special type of the nonlocal setting indicated in the previous section, the so-called Cartan-covering. As mentioned before we shall illustrate this by the $\mathrm{KdV}$-equation.

Let $Y \subset J^{\infty}(x, t ; u)$ be the infinite prolongation of KdV-equation (3). Contact one forms on $T J^{\infty}(x, t ; u)$ are given by

$$
\begin{aligned}
& \alpha_{0}=d u-u_{1} d x-u_{t} d t, \\
& \alpha_{1}=d u_{1}-u_{2} d x-u_{1 t} d t, \\
& \alpha_{2}=d u_{2}-u_{3} d x-u_{2 t} d t .
\end{aligned}
$$

From the total partial derivative operators of the previous section we have

$$
\begin{aligned}
& \widetilde{D}_{x}\left(\alpha_{1}\right)=\alpha_{1}, \quad \widetilde{D}_{x}\left(\alpha_{1}\right)=\alpha_{2}, \ldots, \\
& \widetilde{D}_{t}\left(\alpha_{0}\right)=\alpha_{0} u_{x}+\alpha_{1} u+\alpha_{3}=\alpha_{t}, \\
& \widetilde{D}_{t}\left(\alpha_{i}\right)=\left(\widetilde{D}_{x}\right)^{i}\left(\alpha_{t}\right) .
\end{aligned}
$$

We now define the Cartan-covering of $Y$ by $Y \otimes \mathbb{R}^{\infty}$, where local coordinates are given $\left(x, t, u, u_{1}, \ldots, \alpha_{0}, \alpha_{1}, \ldots\right)$ by

$$
\begin{aligned}
& D_{x}^{C}=\widetilde{D}_{x}+\sum_{i}\left(\alpha_{i+1}\right) \frac{\partial}{\partial \alpha_{i}}, \\
& D_{t}^{C}=\widetilde{D}_{t}+\sum_{i}\left(\widetilde{D}_{x}\right)^{i} \alpha_{t} \frac{\partial}{\partial \alpha_{i}} .
\end{aligned}
$$

It is a straightforward check, and obvious that

$$
\left[D_{x}^{C}, D_{t}^{C}\right]=0
$$

i.e., they form a Cartan distribution on $Y \otimes \mathbb{R}^{\infty}$.

Note 1. Since at first $\alpha_{i}(i=0, \ldots)$ are contact forms, they constitute a Grassmann algebra (graded commutative algebra) $\Lambda(\alpha)$, where

$$
\alpha_{i} \wedge \alpha_{j}=-\alpha_{j} \wedge \alpha_{i}
$$

i.e.,

$$
x y=(-1)^{|x||y|} y x,
$$

where $x, y$ are contact $(*)$-forms of degree $|x|$ and $|y|$ respectively. So in effect we are dealing with a graded covering. 
Note 2. Once we have introduced the Cartan-covering by (18) we can forget about the specifics of $\alpha_{i}(i=0, \ldots)$ and just treat them as (odd) ordinary variables, associated with their differentiation rules.

One can discuss nonlocal symmetries in this type of covering just as in the previous section, the only difference being:

$$
f \in V^{\infty}(Y) \otimes \bigwedge(\alpha) .
$$

In the next section we shall combine constructions of the previous section and this section, in order to construct the recursion operator for symmetries.

\section{THE RECURSION OPERATOR AS SYMMETRY IN THE CARTAN-COVERING}

We shall discuss the recursion operator for symmetries of $\mathrm{KdV}$-equation as a geometrical object, i.e., a symmetry in the Cartan-covering.

Our starting point is the four-dimensional covering of the $\mathrm{KdV}$-equation in $Y \otimes \mathbb{R}^{4}$ where

$$
\begin{aligned}
\bar{D}_{x}= & D_{x}+u \partial_{w_{1}}+\frac{1}{2} u^{2} \partial_{w_{2}}+\left(u^{3}-3 u_{1}^{2}\right) \partial_{w_{3}}+w_{1} \partial_{w_{4}} \\
\bar{D}_{t}= & D_{t}+\left(\frac{1}{2} u^{2}+u_{2}\right) \partial_{w_{1}}+\left(\frac{1}{3} u^{3}-\frac{1}{2} u_{1}^{2}+u u_{2}\right) \partial_{w_{2}} \\
& +\left(\frac{3}{4} u^{4}-6 u_{1} u_{3}+3 u^{2} u_{2}-6 u u_{1}^{2}+3 u_{2}^{2}\right) \partial_{w_{3}}+\left(u_{1}+w_{2}\right) \partial_{w_{4}},
\end{aligned}
$$

$\bar{D}_{x}, \bar{D}_{t}$ satisfy the covering condition (9), and note that due to the fact that the coefficients of $\partial_{w_{i}}(i=1,2,3)$ in (20) are independent of $w_{j}(j=1,2,3)$. These coefficients constitute conservation laws for the KdV-equation. We have the following "formal" variables.

$$
\begin{aligned}
& w_{1}=\int u d x, \\
& w_{2}=\int \frac{1}{2} u^{2} d x, \\
& w_{3}=\int\left(u^{3}-3 u_{1}^{2}\right) d x, \\
& w_{4}=\int w_{1} d x,
\end{aligned}
$$

where in (21) $w_{4}$ is of a higher nonlocality.

We now build the Cartan-covering of the previous section on the covering given by (20) by introduction of the contact forms $\alpha_{0}, \alpha_{1}, \alpha_{2}, \ldots$ (16) and 


$$
\begin{aligned}
& \alpha_{-1}=d w_{1}-u d x-\left(\frac{1}{2} u^{2}+u_{2}\right) d t \\
& \alpha_{-2}=d w_{2}-\frac{1}{2} u^{2} d x-\left(\frac{1}{3} u^{3}-\frac{1}{2} u_{1}^{2}+u u_{2}\right) d t
\end{aligned}
$$

and similarly for $\alpha_{-3}, \alpha_{-4}$. It is straightforward to prove the following relations

$$
\begin{aligned}
& \bar{D}_{x}\left(\alpha_{-1}\right)=\alpha_{0}, \quad \bar{D}_{t}\left(\alpha_{-1}\right)=u \alpha_{0}+\alpha_{0}, \\
& \bar{D}_{x}\left(\alpha_{-2}\right)=u \alpha_{0}, \quad \bar{D}_{t}\left(\alpha_{-2}\right)=u^{2} \alpha_{0}-u_{1} \alpha_{1}+u \alpha_{2}+u_{2} \alpha_{0}, \\
& \bar{D}_{x}\left(\alpha_{-3}\right)=3 u^{2} \alpha_{0}-6 u_{1} \alpha_{1}, \ldots
\end{aligned}
$$

We are now constructing symmetries in this Cartan-covering of KdV-equation which are linear w.r.t. $\alpha_{i}(i=-4, \ldots, 0,1, \ldots)$.

The symmetry condition for $f \in C^{\infty}\left(Y \otimes \mathbb{R}^{4}\right) \otimes \Lambda^{1}(\alpha)$ is just given by (7)

$$
\bar{\ell}_{F}^{C}(f)=0,
$$

which for the $\mathrm{KdV}$ equation results in

$$
\bar{D}_{t}^{C}(f)-u \bar{D}_{x}^{C}(f)-u_{x} f-\left(\bar{D}_{x}^{C}\right)^{3} f=0 .
$$

As solutions of these equations we obtained

$$
\begin{aligned}
f^{0}= & \alpha_{0}, \\
f^{1}= & \left(\frac{2}{3} u\right) \alpha_{0}+\alpha_{2}+\left(\frac{1}{3} u_{1}\right) \alpha_{-1}, \\
f^{2}= & \left(\frac{4}{9} u^{2}+\frac{4}{3} u_{2}\right) \alpha_{0}+\left(2 u_{1}\right) \alpha_{1}+\left(\frac{4}{3} u\right) \alpha_{2}+\alpha_{4} \\
& +\frac{1}{3}\left(u u_{1}+u_{3}\right) \alpha_{-1}+\frac{1}{9}\left(u_{1}\right) \alpha_{-2} .
\end{aligned}
$$

As we mentioned above we are working in effect with form-valued vector fields $\vartheta_{f^{0}}, \vartheta_{f^{1}}, \vartheta_{f^{2}}$. For these objects one can define Frölicher-Nijenhuis and (by contraction) Richardson-Nijenhuis brackets [5,6]. Without going into details, for which the reader is referred to [5], we can construct the contraction of a (generalized) symmetry and a form valued symmetry p.e.

$$
R=\left(\frac{2}{3} u \alpha_{0}+\alpha_{2}+\frac{1}{3} u_{1} \alpha_{-1}\right) \frac{\partial}{\partial u}+\cdots
$$

The contraction being defined by

$$
\left.\left.\left.\left(V_{1}\right\lrcorner R\right)=(V\lrcorner R_{u}\right) \partial_{u}+\bar{D}_{x}^{C}(V\lrcorner R_{u}\right) \partial_{u_{1}}+\cdots .
$$

Start now with

$$
V_{1}=u_{1} \frac{\partial}{\partial u}+u_{2} \frac{\partial}{\partial u_{1}}+\cdots
$$


whose prolongation in the setting $Y \otimes \mathbb{R}^{4}$ is

$$
\begin{aligned}
\bar{V}_{1}= & u_{1} \frac{\partial}{\partial u}+u_{2} \frac{\partial}{\partial u_{1}}+\cdots+u \frac{\partial}{\partial w_{1}}+\frac{1}{2} u^{2} \frac{\partial}{\partial w_{2}} \\
& +\left(u^{3}-3 u_{1}^{2}\right) \frac{\partial}{\partial w_{3}}+w_{1} \frac{\partial}{\partial w_{4}}
\end{aligned}
$$

then

$$
\begin{aligned}
(\bar{V}\lrcorner R) & =\left[\left(\frac{2}{3} u\right) u_{1}+1 \cdot u_{3}+\frac{1}{3} u_{1} \cdot u\right] \frac{\partial}{\partial u}+\cdots \\
& =\left(u_{3}+u u_{1}\right) \frac{\partial}{\partial u}+\cdots=V_{3}
\end{aligned}
$$

and similarly

$$
\left.\left(\bar{V}_{3}\right\lrcorner R\right)=\left(u_{5}+\frac{5}{3} u_{3} u+\frac{10}{3} u_{2} u_{1}+\frac{5}{6} u^{2} u_{1}\right) \frac{\partial}{\partial u}+\cdots=V_{5} .
$$

The result given above means that the well known Lenard recursion operator for symmetries of KdV-equation is represented as a symmetry, $\ni_{f_{1}}$, in the Cartancovering of this equation and in effect is a geometrical object.

\section{The Coupled KdV-MKdV System}

In this section we shall discuss the complete integrability of the KdV-mKdV system $\mathcal{E}$, given in (1), i.e.,

$$
\begin{aligned}
& u_{t}=-u_{3}+6 u u_{1}-3 w w_{3}-3 w_{1} w_{2}+3 u_{1} w^{2}+6 u w w_{1}, \\
& w_{t}=-w_{3}+3 w^{2} w_{1}+3 u w_{1}+3 u_{1} w .
\end{aligned}
$$

In order to demonstrate the complete integrability of this system, we shall construct the recursion operator for symmetries of this coupled system, leading to infinite hierarchies of symmetries and, most probably, of conservation laws.

Due to the very special form of the final results, it seems that integrability of this system, which looks at first glance quite ordinary, has not been discussed before. In order to discuss complete integrability, we shall start to discuss conservation laws in Subsection 4.1 leading to the necessary nonlocal variables.

In Subsection 4.2 we shall discuss local and nonlocal symmetries of the system, while in Subsection 4.3 we construct the recursion operator or deformation [5], by the construction of a symmetry in the Cartan covering of Eq. (31).

\subsection{Conservation laws and nonlocal variables}

Here we shall construct conservation laws for (31) in order to arrive at an Abelian covering of the coupled $\mathrm{KdV}-\mathrm{mKdV}$ system as was shown $\mathrm{KdV}$ equation (2).

So we construct $X=X(x, t, u, \ldots, w \ldots), T=T(x, t, u, \ldots, w \ldots)$ such that 


$$
D_{x}(T)=D_{t}(X)
$$

where $D_{x}, D_{t}$ are defined as the total partial derivative operators on the infinite jetbundle associated to Eq. (31) and in a similar way we construct nonlocal conservation laws by the requirement

$$
\bar{D}_{x}(\bar{T})=\bar{D}_{t}(\bar{X}),
$$

where $\bar{D}_{*}$ is defined as the prolongation of $D_{*}$ towards the covering of the equation by nonlocal variables arising from local conservation laws; moreover $\bar{X}, \bar{T}$ are dependent on local variables $x, t, u, \ldots, w, \ldots$ as well as the already determined nonlocal variables, denoted here by $p_{*}$ or $p_{*, *}$, which are associated to the conservation laws $(X, T)$ by the formal definition

$$
\begin{aligned}
D_{x}\left(p_{*}\right) & =\left(p_{*}\right)_{x}=X, \\
D_{t}\left(p_{*}\right) & =\left(p_{*}\right)_{t}=T .
\end{aligned}
$$

Proceeding in this way, we obtained the following set of nonlocal variables

$$
p_{0,1}, p_{0,2}, p_{1}, p_{1,1}, p_{1,2}, p_{2,1}, p_{3}, p_{3,1}, p_{3,2}, p_{4,1}, p_{5},
$$

where their defining equations are given by

$$
\begin{aligned}
\left(p_{1}\right)_{x}= & u, \\
\left(p_{1}\right)_{t}= & 3 u^{2}+3 u w^{2}-u_{2}-3 w w_{2}, \\
\left(p_{0,1}\right)_{x}= & w \\
\left(p_{0,1}\right)_{t}= & 3 u w+w^{3}-w_{2}, \\
\left(p_{0,2}\right)_{x}= & p_{1}, \\
\left(p_{0,2}\right)_{t}= & -6 p_{3}-u_{1}, \\
\left(p_{1,1}\right)_{x}= & \cos \left(2 p_{0,1}\right) p_{1} w+\sin \left(2 p_{0,1}\right) w^{2}, \\
\left(p_{1,1}\right)_{t}= & \cos \left(2 p_{0,1}\right)\left(3 p_{1} u w+p_{1} w^{3}-p_{1} w_{2}+u w_{1}-u_{1} w-w^{2} w_{1}\right) \\
& +\sin \left(2 p_{0,1}\right)\left(4 u w^{2}+w^{4}-2 w w_{2}+w_{1}^{2}\right), \\
\left(p_{1,2}\right)_{x}= & \cos \left(2 p_{0,1}\right) w^{2}-\sin \left(2 p_{0,1}\right) p_{1} w, \\
\left(p_{1,2}\right)_{t}= & \cos \left(2 p_{0,1}\right)\left(4 u w^{2}+w^{4}-2 w w_{2}+w_{1}^{2}\right) \\
& +\sin \left(2 p_{0,1}\right)\left(-3 p_{1} u w-p_{1} w^{3}+p_{1} w_{2}-u w_{1}+u_{1} w+w^{2} w_{1}\right), \\
\left(p_{2,1}\right)_{x}= & \left(4 \cos \left(2 p_{0,1}\right) p_{1,1} w^{2}-4 \sin \left(2 p_{0,1}\right) p_{1} p_{1,1} w+w\left(p_{1}^{2}-2 u+w^{2}\right)\right) / 2, \\
\left(p_{2,1}\right)_{t}= & \left(4 \cos \left(2 p_{0,1}\right) p_{1,1}\left(4 u w^{2}+w^{4}-2 w w_{2}+w_{1}^{2}\right)\right. \\
& +4 \sin \left(2 p_{0,1}\right) p_{1,1}\left(-3 p_{1} u w-p_{1} w^{3}+p_{1} w_{2}-u w_{1}+u_{1} w+w^{2} w_{1}\right) \\
& +3 p_{1}^{2} u w+p_{1}^{2} w^{3}-p_{1}^{2} w_{2}+2 p_{1} u w_{1}-2 p_{1} u_{1} w-2 p_{1} w^{2} w_{1}-8 u^{2} w \\
& \left.-u w^{3}+2 u w_{2}-2 u_{1} w_{1}+2 u_{2} w+w^{5}+3 w^{2} w_{2}\right) / 2, \\
\left(p_{3}\right)_{x}= & \left(-u^{2}-u w^{2}+w w_{2}\right) / 2,
\end{aligned}
$$




$$
\begin{aligned}
\left(p_{3}\right)_{t}= & \left(-4 u^{3}-9 u^{2} w^{2}+2 u u_{2}-3 u w^{4}+11 u w w_{2}-u w_{1}^{2}-u_{1}^{2}+u_{1} w w_{1}\right. \\
& \left.+4 u_{2} w^{2}+6 w^{3} w_{2}+3 w^{2} w_{1}^{2}-w w_{4}+w_{1} w_{3}-w_{2}^{2}\right) / 2, \\
\left(p_{3,1}\right)_{x}= & \left(\cos \left(2 p_{0,1}\right) w\left(p_{1}^{3}-6 p_{1} u+39 p_{1} w^{2}-24 p_{1,1} p_{1,2} w+12 p_{3}+6 u_{1}\right)\right. \\
& +2 \sin \left(2 p_{0,1}\right) w\left(12 p_{1} p_{1,1} p_{1,2}+18 p_{1} w_{1}+2 w^{3}+3 w_{2}\right) \\
& \left.+6 p_{1,2} w\left(-p_{1}^{2}+2 u-w^{2}\right)\right) / 12 \\
\left(p_{3,2}\right)_{x}= & \left(2 \cos \left(2 p_{0,1}\right) w\left(12 p_{1} p_{1,1} p_{1,2}-18 p_{1} w_{1}-2 w^{3}-3 w_{2}\right)\right. \\
& +\sin \left(2 p_{0,1}\right) w\left(p_{1}^{3}-6 p_{1} u+39 p_{1} w^{2}+24 p_{1,1} p_{1,2} w+12 p_{3}+6 u_{1}\right) \\
& \left.+6 p_{1,1} w\left(-p_{1}^{2}+2 u-w^{2}\right)\right) / 12, \\
\left(p_{4,1}\right)_{x}= & \left(8 \operatorname { c o s } ( 2 p _ { 0 , 1 } ) w \left(p_{1}^{3} p_{1,2}+12 p_{1} p_{1,1}^{2} p_{1,2}-6 p_{1} p_{1,2} u+3 p_{1} p_{1,2} w^{2}\right.\right. \\
& \left.-12 p_{1,1} p_{1,2}^{2} w+18 p_{1,1} u w-4 p_{1,1} w^{3}-6 p_{1,1} w_{2}+12 p_{1,2} p_{3}+6 p_{1,2} u_{1}\right) \\
& +8 \sin \left(2 p_{0,1}\right) w\left(p_{1}^{3} p_{1,1}+12 p_{1} p_{1,1} p_{1,2}^{2}-6 p_{1} p_{1,1} u+3 p_{1} p_{1,1} w^{2}\right. \\
& \left.+12 p_{1,1}^{2} p_{1,2} w+12 p_{1,1} p_{3}+6 p_{1,1} u_{1}-18 p_{1,2} u w+4 p_{1,2} w^{3}+6 p_{1,2} w_{2}\right) \\
& +w\left(-p_{1}^{4}-24 p_{1}^{2} p_{1,1}^{2}-24 p_{1}^{2} p_{1,2}^{2}+12 p_{1}^{2} u-6 p_{1}^{2} w^{2}-48 p_{1} p_{3}\right. \\
& -24 p_{1} u_{1}+48 p_{1,1}^{2} u-24 p_{1,1}^{2} w^{2}+48 p_{1,2}^{2} u-24 p_{1,2}^{2} w^{2} \\
& \left.\left.-60 u^{2}+44 u w^{2}+24 u_{2}-13 w^{4}+6 w w_{2}\right)\right) / 48, \\
\left(p_{5}\right)_{x}= & \left(12 u^{3}+24 u^{2} w^{2}-6 u u_{2}+6 u w^{4}-30 u w w_{2}-3 u_{2} w^{2}-8 w^{3} w_{2}\right. \\
& \left.+6 w w_{4}\right) / 6 .
\end{aligned}
$$

In the previous equations, we skipped explicit formulas for $\left(p_{3,1}\right)_{t},\left(p_{3,2}\right)_{t}$, $\left(p_{4,1}\right)_{t}$, and $\left(p_{5}\right)_{t}$, because they are too massive, though quite important for the setting to be well defined and in order to avoid ambiguities. The reader is referred to [3] for these explicit formulas.

It is quite a striking result that functions $\cos \left(2 p_{0,1}\right), \sin \left(2 p_{0,1}\right)$ appear in the presentation of the conservation laws and their associated nonlocal variables.

We should note that $p_{1}, p_{0,1}, p_{3}, p_{5}$ arise from local conservation laws and we shall call $p_{1}, p_{0,1}, p_{3}, p_{5}$ nonlocalities of first order.

In a similar way we see that $p_{0,2}, p_{1,1}, p_{1,2}$ arise from nonlocal conservation laws, where their $x$ - and $t$-derivatives are dependent on the first-order nonlocalities. For this reason $p_{0,2}, p_{1,1}, p_{1,2}$ are called nonlocalities of second order. Proceeding in this way $p_{2,1}, p_{3,1}, p_{3,2}, p_{4,1}$ constitute nonlocalities of third order.

\subsection{Local and nonlocal symmetries}

In this section we shall present results for the construction of local and nonlocal symmetries of system (31). In order to construct these symmetries, we consider the system of partial differential equations obtained by the infinite prolongation of (31) together with the covering by the nonlocal variables 
So, in the augmented setting governed by (31), their total derivatives and the equations given in Subsection 4.1 we construct symmetries $Y=\left(Y^{u}, Y^{w}\right)$ which have to satisfy the symmetry condition

$$
\bar{\ell}_{\mathcal{E}} Y=0 \text {. }
$$

From this condition we obtained the following symmetries

$$
Y_{0,1}, Y_{1,1}, Y_{1,2}, Y_{1,3}, Y_{2,1}, Y_{3,1}, Y_{3,2}, Y_{3,3} \text {, }
$$

where generating functions $Y_{*, *}^{u}, Y_{*, *}^{w}$ are given as

$$
\begin{aligned}
& Y_{0,1}^{u}=3 t\left(6 u u_{1}+6 u w w_{1}+3 u_{1} w^{2}-u_{3}-3 w w_{3}-3 w_{1} w_{2}\right)+x u_{1}+2 u, \\
& Y_{0,1}^{w}=3 t\left(3 u w_{1}+3 u_{1} w+3 w^{2} w_{1}-w_{3}\right)+x w_{1}+w, \\
& Y_{1,1}^{u}=u_{1} \text {, } \\
& Y_{1,1}^{w}=w_{1}, \\
& Y_{1,2}^{u}=\cos \left(2 p_{0,1}\right)\left(2 u w-w_{2}\right)+\sin \left(2 p_{0,1}\right)\left(u_{1}+2 w w_{1}\right), \\
& Y_{1,2}^{w}=-\cos \left(2 p_{0,1}\right) u-\sin \left(2 p_{0,1}\right) w_{1} \text {, } \\
& Y_{1,3}^{u}=\cos \left(2 p_{0,1}\right)\left(u_{1}+2 w w_{1}\right)+\sin \left(2 p_{0,1}\right)\left(-2 u w+w_{2}\right), \\
& Y_{1,3}^{w}=-\cos \left(2 p_{0,1}\right) w_{1}+\sin \left(2 p_{0,1}\right) u, \\
& Y_{2,1}^{u}=\left(2 \cos \left(2 p_{0,1}\right)\left(p_{1,1} u_{1}+2 p_{1,1} w w_{1}-2 p_{1,2} u w+p_{1,2} w_{2}\right)\right. \\
& +2 \sin \left(2 p_{0,1}\right)\left(-2 p_{1,1} u w+p_{1,1} w_{2}-p_{1,2} u_{1}-2 p_{1,2} w w_{1}\right) \\
& \left.+2 p_{1} u w-p_{1} w_{2}+2 u w_{1}+3 u_{1} w+2 w^{2} w_{1}-w_{3}\right) / 2 \text {, } \\
& Y_{2,1}^{w}=\left(2 \cos \left(2 p_{0,1}\right)\left(-p_{1,1} w_{1}+p_{1,2} u\right)+2 \sin \left(2 p_{0,1}\right)\left(p_{1,1} u+p_{1,2} w_{1}\right)\right. \\
& \left.-p_{1} u+u_{1}+w w_{1}\right) / 2 \text {, } \\
& Y_{3,1}^{u}=\left(6 u u_{1}+6 u w w_{1}+3 u_{1} w^{2}-u_{3}-3 w w_{3}-3 w_{1} w_{2}\right) / 3, \\
& Y_{3,1}^{w}=\left(3 u w_{1}+3 u_{1} w+3 w^{2} w_{1}-w_{3}\right) / 3, \\
& Y_{3,2}^{u}=\left(\operatorname { c o s } ( 2 p _ { 0 , 1 } ) \left(-2 p_{1}^{2} u w+p_{1}^{2} w_{2}-4 p_{1} u w_{1}-6 p_{1} u_{1} w-4 p_{1} w^{2} w_{1}+2 p_{1} w_{3}\right.\right. \\
& +8 p_{1,1} p_{1,2} u_{1}+16 p_{1,1} p_{1,2} w w_{1}-8 p_{1,2}^{2} u w+4 p_{1,2}^{2} w_{2}-4 p_{2,1} u_{1} \\
& -8 p_{2,1} w w_{1}+10 u^{2} w+6 u w^{3}-8 u w_{2}-14 u_{1} w_{1}-8 u_{2} w-11 w^{2} w_{2} \\
& \left.-14 w w_{1}^{2}+2 w_{4}\right)+2 \sin \left(2 p_{0,1}\right)\left(-8 p_{1,1} p_{1,2} u w+4 p_{1,1} p_{1,2} w_{2}-2 p_{1,2}^{2} u_{1}\right. \\
& -4 p_{1,2}^{2} w w_{1}+4 p_{2,1} u w-2 p_{2,1} w_{2}+6 u u_{1}+10 u w w_{1}+3 u_{1} w^{2}-u_{3} \\
& \left.+2 w^{3} w_{1}-3 w w_{3}-5 w_{1} w_{2}\right)+4 p_{1,2}\left(2 p_{1} u w-p_{1} w_{2}+2 u w_{1}+3 u_{1} w\right. \\
& \left.\left.+2 w^{2} w_{1}-w_{3}\right)\right) / 8 \text {, } \\
& Y_{3,2}^{w}=\left(\operatorname { c o s } ( 2 p _ { 0 , 1 } ) \left(p_{1}^{2} u-2 p_{1} u_{1}-2 p_{1} w w_{1}-8 p_{1,1} p_{1,2} w_{1}+4 p_{1,2}^{2} u+4 p_{2,1} w_{1}\right.\right. \\
& \left.-4 u^{2}-3 u w^{2}+2 u_{2}+4 w w_{2}+2 w_{1}^{2}\right) \\
& +2 \sin \left(2 p_{0,1}\right)\left(4 p_{1,1} p_{1,2} u+2 p_{1,2}^{2} w_{1}-2 p_{2,1} u-3 u w_{1}-3 u_{1} w-3 w^{2} w_{1}\right. \\
& \left.\left.+w_{3}\right)+4 p_{1,2}\left(-p_{1} u+u_{1}+w w_{1}\right)\right) / 8 \text {, }
\end{aligned}
$$




$$
\begin{aligned}
Y_{3,3}^{u}= & \left(2 \operatorname { c o s } ( 2 p _ { 0 , 1 } ) \left(2 p_{1,1}^{2} u_{1}+4 p_{1,1}^{2} w w_{1}-4 p_{2,1} u w+2 p_{2,1} w_{2}-6 u u_{1}\right.\right. \\
& \left.-10 u w w_{1}-3 u_{1} w^{2}+u_{3}-2 w^{3} w_{1}+3 w w_{3}+5 w_{1} w_{2}\right) \\
& +\sin \left(2 p_{0,1}\right)\left(-2 p_{1}^{2} u w+p_{1}^{2} w_{2}-4 p_{1} u w_{1}-6 p_{1} u_{1} w-4 p_{1} w^{2} w_{1}+2 p_{1} w_{3}\right. \\
& -8 p_{1,1}^{2} u w+4 p_{1,1}^{2} w_{2}-4 p_{2,1} u_{1}-8 p_{2,1} w w_{1}+10 u^{2} w+6 u w^{3} \\
& \left.-8 u w_{2}-14 u_{1} w_{1}-8 u_{2} w-11 w^{2} w_{2}-14 w w_{1}^{2}+2 w_{4}\right) \\
& \left.+4 p_{1,1}\left(2 p_{1} u w-p_{1} w_{2}+2 u w_{1}+3 u_{1} w+2 w^{2} w_{1}-w_{3}\right)\right) / 8 \\
Y_{3,3}^{w}= & \left(2 \cos \left(2 p_{0,1}\right)\left(-2 p_{1,1}^{2} w_{1}+2 p_{2,1} u+3 u w_{1}+3 u_{1} w+3 w^{2} w_{1}-w_{3}\right)\right. \\
& +\sin \left(2 p_{0,1}\right)\left(p_{1}^{2} u-2 p_{1} u_{1}-2 p_{1} w w_{1}+4 p_{1,1}^{2} u+4 p_{2,1} w_{1}-4 u^{2}\right. \\
& \left.\left.-3 u w^{2}+2 u_{2}+4 w w_{2}+2 w_{1}^{2}\right)+4 p_{1,1}\left(-p_{1} u+u_{1}+w w_{1}\right)\right) / 8 .
\end{aligned}
$$

\subsection{Recursion operator}

Here we present the recursion operator $\mathcal{R}$ for symmetries for this case obtained as a higher symmetry in the Cartan covering of system of Eqs. (1) augmented by equations governing the nonlocal variables (34).

As demonstrated there, this symmetry is a form-valued vector field (or a vectorfield-valued one-form) and has to satisfy

$$
\bar{\ell}_{\mathcal{E}}^{C} \mathcal{R}=0 .
$$

In order to arrive at a nontrivial result as was explained for classical $\mathrm{KdV}$ equation (3), (25), we have to introduce nonlocal variables

$p_{0,1}, p_{0,2}, p_{1}, p_{1,1}, p_{1,2}, p_{2,1}, p_{3}, p_{3,1}, p_{3,2}, p_{4,1}, p_{5}$

and their associated Cartan contact forms

$$
\omega_{p_{0,1}}, \omega_{p_{0,2}}, \omega_{p_{1}}, \omega_{p_{1,1}}, \omega_{p_{1,2}}, \omega_{p_{2,1}}, \omega_{p_{3}}, \omega_{p_{3,1}}, \omega_{p_{3,2}}, \omega_{p_{4,1}}, \omega_{p_{5}} .
$$

The final result, which is dependent on the nonlocal Cartan forms

$$
\omega_{p_{0,1}}, \omega_{p_{1}}, \omega_{p_{1,1}}, \omega_{p_{1,2}},
$$

is given by

$$
\mathcal{R}=R^{u} \frac{\partial}{\partial u}+R^{w} \frac{\partial}{\partial w}+\cdots,
$$

where the components $R^{u}, R^{w}$ are given by

$$
\begin{aligned}
R_{u}= & \omega_{u_{2}}(-1)+\omega_{u}\left(4 u+w^{2}\right)+\omega_{w_{2}}(-2 w)+\omega_{w_{1}}\left(-w_{1}\right)+\omega_{w}\left(3 u w-2 w_{2}\right) \\
& +\omega_{p_{1,2}}\left(-\cos \left(2 p_{0,1}\right)\left(u_{1}+2 w w_{1}\right)+\sin \left(2 p_{0,1}\right)\left(2 u w-w_{2}\right)\right) \\
& +\omega_{p_{1,1}}\left(\cos \left(2 p_{0,1}\right)\left(-2 u w+w_{2}\right)-\sin \left(2 p_{0,1}\right)\left(u_{1}+2 w w_{1}\right)\right)
\end{aligned}
$$




$$
\begin{aligned}
& +\omega_{p_{1}}\left(2 u_{1}+w w_{1}\right)+\omega_{p_{0,1}}\left(2 p_{1} u w-p_{1} w_{2}+2 u w_{1}+3 u_{1} w+2 w^{2} w_{1}-w_{3}\right), \\
R_{w}= & \omega_{w_{2}}(-1)+\omega_{w}\left(2 u+w^{2}\right)+\omega_{u}(2 w) \\
& +\omega_{p_{1,2}}\left(\cos \left(2 p_{0,1}\right) w_{1}-\sin \left(2 p_{0,1}\right) u\right) \\
& +\omega_{p_{1,1}}\left(\cos \left(2 p_{0,1}\right) u+\sin \left(2 p_{0,1}\right) w_{1}\right) \\
& +\omega_{p_{1}}\left(w_{1}\right)+\omega_{p_{0,1}}\left(-p_{1} u+u_{1}+w w_{1}\right) .
\end{aligned}
$$

We shall now present this result in a more conventional form which appeals to expressions using operators of the form $D_{x}$ and $D_{x}^{-1}$. In order to do this, we first split (37) into the so-called local part and nonlocal parts, consisting of terms associated to $\omega_{u_{2}}, \omega_{u}, \omega_{w_{2}}, \omega_{w_{1}}, \omega_{w}$ and those associated to $\omega_{p_{1,2}}, \omega_{p_{1,1}}, \omega_{p_{1}}, \omega_{p_{0,1}}$ respectively. The first part will account for $D_{x}$ presentation, while the second one accounts for the $D_{x}^{-1}$ part.

Due to the action of contraction $\left.\ni_{\varphi}\right\lrcorner \mathcal{R}$, the local part is given by the following matrix operator:

$$
\left[\begin{array}{cc}
-D_{x}^{2}+4 u+w^{2} & -2 w D_{x}^{2}-w_{1} D_{x}+3 u w-2 w_{2} \\
2 w & -D_{x}^{2}+2 u+w^{2}
\end{array}\right]
$$

The nonlocal part will be split into parts associated to $\omega_{p_{1}}, \omega_{p_{0,1}}$ and $\omega_{p_{1,2}}, \omega_{p_{1,1}}$, respectively. The first one is given as

$$
\left[\begin{array}{cc}
\left(2 u_{1}+w w_{1}\right) D_{x}^{-1} & \left(2 p_{1} u w-p_{1} w_{2}+2 u w_{1}+3 u_{1} w+2 w^{2} w_{1}-w_{3}\right) D_{x}^{-1} \\
w_{1} D_{x}^{-1} & \left(-p_{1} u+u_{1}+w w_{1}\right) D_{x}^{-1}
\end{array}\right] .
$$

To deal with the last part, let us introduce the notation:

$$
\begin{aligned}
& A_{1}=\cos \left(2 p_{0,1}\right)\left(-2 u w+w_{2}\right)-\sin \left(2 p_{0,1}\right)\left(u_{1}+2 w w_{1}\right), \\
& A_{2}=\cos \left(2 p_{0,1}\right) u+\sin \left(2 p_{0,1}\right) w_{1}, \\
& B_{1}=-\cos \left(2 p_{0,1}\right)\left(u_{1}+2 w w_{1}\right)+\sin \left(2 p_{0,1}\right)\left(2 u w-w_{2}\right), \\
& B_{2}=\cos \left(2 p_{0,1}\right) w_{1}-\sin \left(2 p_{0,1}\right) u
\end{aligned}
$$

being the coefficients at $\omega_{p_{1,1}}$ and $\omega_{p_{1,2}}$ in (37).

According to the presentations of $\left(p_{1,1}\right)_{x}$ and $\left(p_{1,2}\right)_{x}$, i.e.,

$$
\begin{aligned}
& \left(p_{1,1}\right)_{x}=\cos \left(2 p_{0,1}\right) p_{1} w+\sin \left(2 p_{0,1}\right) w^{2}, \\
& \left(p_{1,2}\right)_{x}=\cos \left(2 p_{0,1}\right) w^{2}-\sin \left(2 p_{0,1}\right) p_{1} w,
\end{aligned}
$$

we introduce their partial derivatives with respect to $p_{0,1}, p_{1}$, and $w$ as

$$
\begin{aligned}
& \alpha_{1}=-2 p_{1} w \sin \left(2 p_{0,1}\right)+2 w^{2} \cos \left(2 p_{0,1}\right), \\
& \alpha_{2}=w \cos \left(2 p_{0,1}\right), \\
& \alpha_{3}=p_{1} \cos \left(2 p_{0,1}\right)+2 w \sin \left(2 p_{0,1}\right), \\
& \beta_{1}=-2 w^{2} \sin \left(2 p_{0,1}\right)-2 p_{1} w \cos \left(2 p_{0,1}\right),
\end{aligned}
$$




$$
\begin{aligned}
& \beta_{2}=-w \sin \left(2 p_{0,1}\right), \\
& \beta_{3}=2 w \cos \left(2 p_{0,1}\right)-p_{1} \sin \left(2 p_{0,1}\right) .
\end{aligned}
$$

From this we arrive in a straightforward way at the last nonlocal part of the recursion operator, i.e.,

$$
\begin{aligned}
& {\left[\begin{array}{ll}
A_{1} D_{x}^{-1} \alpha_{2} D_{x}^{-1} & A_{1} D_{x}^{-1}\left(\alpha_{1} D_{x}^{-1}+\alpha_{3}\right) \\
A_{2} D_{x}^{-1} \alpha_{2} D_{x}^{-1} & A_{2} D_{x}^{-1}\left(\alpha_{1} D_{x}^{-1}+\alpha_{3}\right)
\end{array}\right]} \\
& \quad+\left[\begin{array}{cc}
B_{1} D_{x}^{-1} \beta_{2} D_{x}^{-1} & B_{1} D_{x}^{-1}\left(\beta_{1} D_{x}^{-1}+\beta_{3}\right) \\
B_{2} D_{x}^{-1} \beta_{2} D_{x}^{-1} & B_{2} D_{x}^{-1}\left(\beta_{1} D_{x}^{-1}+\beta_{3}\right)
\end{array}\right] .
\end{aligned}
$$

So, in the final form we obtain the recursion operator as

$$
\begin{aligned}
\mathcal{R} & =\left[\begin{array}{cc}
-D_{x}^{2}+4 u+w^{2} & -2 w D_{x}^{2}-w_{1} D_{x}+3 u w-2 w_{2} \\
2 w & -D_{x}^{2}+2 u+w^{2}
\end{array}\right] \\
& +\left[\begin{array}{cc}
\left(2 u_{1}+w w_{1}\right) D_{x}^{-1} & \left(2 p_{1} u w-p_{1} w_{2}+2 u w_{1}+3 u_{1} w+2 w^{2} w_{1}-w_{3}\right) D_{x}^{-1} \\
w_{1} D_{x}^{-1} & \left(-p_{1} u+u_{1}+w w_{1}\right) D_{x}^{-1}
\end{array}\right] \\
& +\left[\begin{array}{cc}
A_{1} D_{x}^{-1} \alpha_{2} D_{x}^{-1} & A_{1} D_{x}^{-1}\left(\alpha_{1} D_{x}^{-1}+\alpha_{3}\right) \\
A_{2} D_{x}^{-1} \alpha_{2} D_{x}^{-1} & A_{2} D_{x}^{-1}\left(\alpha_{1} D_{x}^{-1}+\alpha_{3}\right)
\end{array}\right] \\
& +\left[\begin{array}{cc}
B_{1} D_{x}^{-1} \beta_{2} D_{x}^{-1} & B_{1} D_{x}^{-1}\left(\beta_{1} D_{x}^{-1}+\beta_{3}\right) \\
B_{2} D_{x}^{-1} \beta_{2} D_{x}^{-1} & B_{2} D_{x}^{-1}\left(\beta_{1} D_{x}^{-1}+\beta_{3}\right)
\end{array}\right] .
\end{aligned}
$$

\section{CONCLUSION}

We gave an outline of the theory of symmetries of differential equations, leading to the construction of recursion operators for symmetries of such equations. The extension of this theory to the nonlocal setting of differential equations is essential for getting nontrivial results. The theory has been applied to the construction of the recursion operator for symmetries for a coupled $\mathrm{KdV}-\mathrm{mKdV}$ system, leading to a highly nonlocal result for this system. Moreover the appearance of nonpolynomial nonlocal terms in all results, e.g., conservation laws, symmetries and recursion operator is striking and reveals some unknown and intriguing underlying structure of the equations. Work on the construction of Bäcklund transformations for this system is in progress.

\section{REFERENCES}

[1] Dodd R.K., Eilbeck J.C., Gibbons J.D., Morris H.C. - Solitons and Nonlinear Wave Equations, Academic Press, 1982.

[2] Kersten P.H.M. - Supersymmetries and recursion operators for $N=2$ supersymmetric KdV-equation, RIMS Kokyuroku 1150 (2000) 153-161.

[3] Kersten P.H.M., Krasil'shchik I.S. - Complete integrability of the soupled KdV-mKdV system, in: Marimoto T., Sato H., Yamaguchi K. (Eds.), Lie Groups, Geometric Structures and 
Differential Equations - One Hundred Years after Sophus Lie, in: Adv. Stud. Pure Math., vol. 37, Math. Soc. Japan, 2002, pp. 151-171, arXiv:nlin.SI/0010041(25-10-2000).

[4] Khor'kova N.G. - Conservation laws and nonlocal symmetries, Mat. Zametki 44 (1) (1988) 134 144, 157, translation in Math. Notes 44 (1-2) (1988) 562-568.

[5] Krasil'shchik I.S. - Some new cohomological invariants for nonlinear differential equations, Differential Geom. Appl. 2 (4) (1992) 307-350.

[6] Krasil'shchik I.S., Kersten P.H.M. - Symmetries and Recursion Operators for Classical and Supersymmetric Differential Equations, Kluwer Acad. Publ., Dordrecht, 2000.

[7] Krasil'shchik I.S., Lychagin V.V., Vinogradov A.M. - Geometry of Jet Spaces and Nonlinear Partial Differential Equations, Gordon and Breach, New York, 1986.

[8] Krasil'shchik I.S., Vinogradov A.M. - Nonlocal trends in the geometry of differential equations: Symmetries, conservation laws, and Bäcklund transformations, Acta Appl. Math. 15 (1-2) (1989) 161-209.

[9] Krivonos S., Sorin A. - Extended $N=2$ supersymmetric matrix $(1, s)-\mathrm{KdV}$ hierarchies, Phys. Lett. A 251 (1999) 109.

[10] Mathieu P. - Open problems for the super KdV equation, in: AARMS-CRM Workshop on Baecklund and Darboux transformations. The Geometry of Soliton Theory (June 4-9, 1999, Halifax, Nova Scotia).

[11] Vinogradov A.M. - Local symmetries and conservation laws, Acta Appl. Math. 3 (1984) 21-78. 